Lexis Vol. XLII (2) 2018: 273-326

\title{
Desarrollo de la competencia léxica a través de estrategias de aprendizaje de vocabulario en aprendientes" de inglés como lengua extranjera
}

\author{
Cristián Sanhueza C. \\ Anita Ferreira C. \\ Katia Sáez C. \\ Universidad de Concepción
}

\section{RESUMEN}

Este estudio se circunscribe en el ámbito de la lingüística aplicada y su objetivo es determinar el grado de incidencia que tiene el uso de estrategias de aprendizaje en el desarrollo léxico. Para esto se diseñó una investigación cuasi-experimental con pre y postest y la implementación de 6 estrategias de aprendizaje de vocabulario como tratamiento. La medición se realizó a través de las pruebas de niveles de vocabulario (VLT) y la prueba de Perfil de Frecuencia Léxica (LFP). Los resultados muestran que el proceso de intervención léxica sí tuvo efectos positivos en los participantes del grupo

"Este artículo adscribe al uso del término aprendiente (en inglés, learner), definido por el Instituto Cervantes como "la persona que se encuentra en proceso de aprendizaje de una lengua extranjera, al margen de otras consideraciones, como la edad o el contexto en que aprende" (Centro Virtual Cervantes 2015). Para una lectura detallada del concepto ver Consejo de Europa (2001). Marco común europeo de referencia para las lenguas: aprendizaje, enseñanza, evaluación. Madrid: Instituto Cervantes - Ministerio de Educación, Cultura y Deporte. 
experimental en las tres pruebas en relación a los del grupo de control, siendo el conocimiento receptivo el que más progresa.

Palabras clave: competencia léxica, conocimiento receptivo, conocimiento productivo, uso léxico

\section{ABSTRACT}

This study is circumscribed by the field of applied linguistics and its aim is to determine the effects of learning strategies on the participants' lexical competence. For this, a quasi-experimental study with pre and post test and the implementation of 6 learning strategies as treatment were designed. Measurements were performed using the Vocabulary Levels Test and the Lexical Frequency Profile. Results showed that the treatment did have a positive effect on the experimental group in all the tests in relation to the control group, being receptive knowledge the one that showed more progress. Keywords: lexical competence, receptive knowledge, productive knowledge, lexical use

\section{Introducción}

La investigación en la adquisición de vocabulario ha tomado un lugar preponderante en la indagación lingüística en las últimas tres décadas. Hasta los años ochenta, la gramática era el área de estudio central en el ámbito de la Lingüística Aplicada. Hoy en día, la adquisición y aprendizaje del vocabulario han demostrado ser un elemento clave en el desarrollo de la Interlengua (Albrechtsen, Haastrup y Henriksen 2008). En este sentido, está ampliamente establecido que las habilidades de comprensión se desarrollan gracias al conocimiento receptivo que el sujeto posee. Las habilidades productivas, por su parte, hacen lo propio gracias al conocimiento productivo o, en otras palabras, al uso activo del conocimiento receptivo. Este despliegue activo del conocimiento receptivo, no solo gramatical, sino principalmente léxico, es el que presenta desafíos interesantes de abordar investigativamente con el propósito de descubrir cuál es la mejor manera de realizar esta transferencia de conocimiento. 
La competencia léxica puede desarrollarse desde niveles superficiales a más profundos en las diferentes etapas de aprendizaje (Nation y Meara 2010; Laufer y Goldstein 2004; Laufer 1998, Read 1993). Aun así, se considera que el volumen del conocimiento léxico, por sobre su profundidad, es de crucial importancia para los aprendientes. Igualmente, se ha establecido que el volumen del conocimiento léxico es un buen predictor de la comprensión lectora (Koda 1989; Coady 1993) y que se correlaciona bien con la calidad de la escritura (Linnarud 1986; Astika, 1993) y la fluidez (Towell, Hawkins y Bazergui 1998; Luoma 2004). Además, los aprendientes asocian su progreso en el aprendizaje de la lengua al incremento en el número de palabras que conocen (Laufer 1998).

En este estudio se propone examinar el desarrollo de tres componentes relativos a la competencia léxica. El conocimiento receptivo básico, es decir, la comprensión del significado más frecuente o base de una palabra utilizando la prueba VLT (Vocabulary Levels Test) (Nation 1983, 1990, 2001) y, además, dos tipos de conocimiento productivo, controlado y libre. El primero conlleva la producción de una palabra inducida por una tarea; para esto se utilizará la prueba VLT en su formato productivo. Y el segundo, busca el uso de acuerdo a la voluntad del aprendiente, sin una tarea que induzca el uso específico de alguna palabra, como es el caso de la composición libre, para esto se utilizó la prueba de Perfil de Frecuencia Léxica (Lexical Frequency Profile - LFP) (Laufer y Nation 1995). Estas mediciones se realizaron en forma de pretest a los participantes del estudio. Posteriormente, se implementó un proceso de intervención en el que se utilizaron estrategias y técnicas que posibilitaron el uso léxico durante un período de dos semestres académicos. Seguidamente, se aplicaron nuevamente los instrumentos antes mencionados a modo de postest en su versión equivalente, con el propósito de determinar el progreso que hubo durante dicho período, manifestado a través del crecimiento en el conocimiento léxico receptivo, productivo controlado y en el uso libre del vocabulario. 


\section{La competencia léxica}

Los primeros estudios que se reportaron en esta materia (Yoshida 1978; Cohen y Aphek 1981; Palmberg 1987; Luppescu y Day, 1993; Hulstijin, Hollander y Greidanus 1996) normalmente investigaban la adquisición de palabras que habían sido específicamente seleccionadas para el experimento y no los cambios en el volumen global del vocabulario. Esto pudo haberse debido, principalmente, a la dificultad que suponía una conceptualización certera del conocimiento léxico en L2 y el diseño de instrumentos válidos y confiables que midieran este conocimiento. Aunque los investigadores defieren en su definición de conocimiento léxico, existe el consenso que éste no es un fenómeno dicotómico, sino gradual.

Por tanto, una preocupación central en la investigación del ámbito léxico ha sido la construcción de una definición más exacta de la competencia léxica como constructo. Una contribución en este sentido han sido las dimensiones de amplitud (breath) y profundidad (depth) que han sido estudiadas extensamente (Nation 1983, 1990, 2001; Laufer 1998, 2013; Laufer y Nation 1999; Laufer y Goldstein 2004; Schmitt, Schmitt y Clapham 2001). Un vocabulario amplio, con conocimiento de palabras a través de una variedad de bandas de frecuencia, es una característica esencial de un buen manejo léxico. Es decir, la competencia léxica se define, predominantemente, por el número de palabras que posee el aprendiente. Conjuntamente, se ha determinado que un uso léxico efectivo en la escritura ejerce una influencia positiva en la medición de su calidad y en la proficiencia general del aprendiente (Lee 2003; Llach y Gallego 2009; Morris y Cobb 2004). Por su parte, los aprendientes mencionan al conocimiento de vocabulario como un factor crucial en el desarrollo de la escritura (Leki y Carson 1994; Polio y Glew 1996; Laufer 2005; Laufer y Goldstein 2004). No es sorpresivo, entonces, que el interés por precisar la importancia de la competencia léxica sea de tanta relevancia. 


\section{1. ¿Qué se entiende por conocimiento léxico?}

En términos de Nation, "las palabras no son unidades lingüísticas aisladas, sino, unidades interrelacionadas con sistemas y niveles" (2001: 23). Debido a esto, dice el autor, hay muchos elementos por conocer acerca de la palabra, pues existen variados niveles de conocimiento. En este sentido, uno de los principales enfoques en torno al conocimiento léxico es la relación entre el aprendizaje de ítems individuales y los sistemas de aprendizaje. Es posible, entonces, aprender a reconocer una palabra solo memorizándola $\mathrm{o}$, por otro lado, reconociendo la forma regular en que se deletrea una palabra al aprender el sistema que la rige, tanto desde el punto vista fonológico como del ortográfico. Esta relación del conocimiento del ítem y el conocimiento del sistema es compleja y ha habido enorme debate al respecto. En este sentido, será útil la puntualización de algunos conceptos ampliamente utilizados en el estudio de la adquisición y el aprendizaje de vocabulario que nos ayudarán a comprender mejor la naturaleza de la palabra.

Una de las distinciones básicas que es necesario realizar es la que ocurre entre dos conceptos ampliamente usados en el ámbito léxico como lo son el de tipo (type) y el de caso (token). El concepto de caso se refiere al número total de palabras de un texto, incluyendo las palabras que se usen más de una vez. Por el contrario, el concepto de tipo corresponde al número total de palabras diferentes, es decir, aquellas palabras del texto que son utilizadas por única vez. Por ejemplo, cuando un profesor asigna la tarea de escribir un texto de trescientas palabras, el profesor y los alumnos entienden que tal cantidad se refiere al número de casos, muchos de los cuales son utilizados reiteradamente, por ejemplo, the, $a, t o$, and, in, that, aun cuando el número de tipos sean considerablemente inferior a trescientos. La proporción relativa entre tipo y caso, conocida como la relación tipo-caso (type-token ratio), es una medida ampliamente utilizada, tanto en el desarrollo de la L2 como de la lengua materna.

Palabras como las que se dan en el ejemplo anterior llevan a preguntarnos si se les debe considerar como palabras de vocabulario. Este tipo de palabras, artículos, preposiciones, pronombres, 
conjunciones, auxiliares, etc., constituyen las denominadas palabras funcionales y se las considera más pertenecientes al área gramatical que a la del vocabulario. A diferencia de las palabras de contenido, sustantivos, verbos, adjetivos y adverbios, las palabras funcionales poseen poco a nada de significado aislado, y más bien, sirven propósitos, principalmente, gramaticales. En términos generales, cuando nos proponemos medir el significado, nos centramos en el conocimiento de las palabras de contenido. Read (2000) comenta que aun cuando solo nos restrinjamos a las palabras de contenido, igual existe el problema de la variedad de las formas en las que se presentan están palabras. Por ejemplo, tomemos la palabra base wait (esperar), también existen las formas waits, waited y waiting. Del mismo modo palabras como society, societies, society's y societies'. En ambas instancias, consideraríamos a estas palabras como formas de una misma palabra. Gramaticalmente, lo que sucede es que se adosa un sufijo a la forma base sin cambiar el significado ni la clase de la palabra. En el ámbito léxico, la forma base y sus inflexiones se conocen como lema. Por tanto, cuando se requiere medir el conocimiento léxico y se realiza un conteo de tipos de palabras, tanto de textos escritos como orales, una de las consideraciones para llevar a cabo este proceso es lematizar los casos, con el propósito de que las formas flexionadas sean contadas como instancias de un mismo lema como forma base.

No obstante, las formas base no solo dan lugar a las inflexiones, sino también a las derivaciones, que comúnmente modifican la categoría de la palabra y adicionan otros elementos a su significado. Un ejemplo de esto podría ser la palabra inglesa leak (gotear), con sus formas flexionadas leaks, leaking, leaked y sus formas derivadas leaky, leakiness, leakage y leaker. Todas estas palabras están estrechamente relacionadas en forma y significado. Este grupo de formas, derivadas y flexionadas, que comparten un significado común es conocido como familia de palabras. Nation (2001) reconoce que uno de los principales problemas cuando se utiliza esta medida para contabilizar palabras es decidir qué se debe incluir en una familia de palabras y qué no. En este sentido, este autor explica que, por ejemplo, el conocimiento de prefijos y sufijos de 
los aprendientes se desarrolla a medida que adquieren más experiencia en la L2. Por consiguiente, lo que pudiera ser una familia de palabras adecuada para un aprendiente pudiera estar lejos del nivel de proficiencia actual de otro aprendiente. De ahí, que sea necesario utilizar una escala de familias de palabras, desde el nivel más elemental y transparente hacia niveles más complejos y menos obvios como se establece en las bandas de frecuencia.

\subsection{Distinción entre conocimiento receptivo y productivo}

Varios investigadores en el ámbito léxico distinguen entre el conocimiento pasivo (receptivo) y activo (productivo) de una palabra (Meara 1990; Nation 2001). El conocimiento pasivo se asocia, normalmente, a la habilidad auditiva y lectora e implica que el aprendiente es capaz de comprender este input. En el vocabulario, esto significa que somos capaces de percibir la forma de la palabra y recuperar su significado o significados. Por el contrario, el conocimiento activo está asociado a la habilidad de escritura y habla e implica que podemos recuperar la forma hablada o escrita apropiada del significado que queremos expresar. Aun así, la distinción entre conocimiento pasivo y activo no es tan simple como estas asociaciones suponen. Read (2000) señala que, como resultado de diferentes distinciones hechas por diferentes investigadores, es complejo realizar comparaciones entre estudios de estos dos conceptos.

Otro elemento problemático al hacer la distinción entre conocimiento pasivo y activo es que no existe consenso sobre si la relación que existe entre ambos es dicotómica o continua. Teichroew (1982) sugiere que esta relación es arbitraria y propone abordar los conceptos de activo y pasivo como polos de un continuo que representa grados crecientes de conocimiento. Por su parte, Meara (1990) cree que estos dos tipos de conocimiento constituyen tipos de conocimiento asociativo fundamentalmente diferente: el conocimiento activo opera por el contacto con otras palabras en la lengua, mientras que el conocimiento pasivo puede activarse solamente por un estímulo externo. Nation (2001) critica esta visión planteando que el uso de la lengua no está únicamente motivado por su carácter asociativo, 
sino, por el significado. El ser capaz de nombrar un objeto usando la lengua meta puede ser estimulado externamente al ver tal objeto sin que, necesariamente, se activen vínculos asociativos con otras palabras de la L2. Laufer y Goldstein (2004) plantean, por su parte, que una palabra que se usa correctamente por parte del aprendiente, será entendida por éste cuando la lee o la escucha. Sin embargo, lo opuesto no necesariamente ocurre. En opinión de Laufer (1998) la mera memorización de la palabra en un contexto determinado, sin la comprensión del significado, no puede considerarse conocimiento productivo. De este modo, se puede considerar que el vocabulario receptivo es más amplio que el vocabulario activo.

A pesar de los diferentes enfoques, todos coinciden en el amplio uso de ambas dimensiones del conocimiento léxico, ya sea desde una perspectiva dicotómica o multicomponencial. Es así que los términos receptivos y productivos se aplican a una variedad de tipos de conocimientos y usos lingüísticos. Cuando se refieren al vocabulario, estos términos cubren todos los aspectos que estarían involucrados en el conocimiento de una palabra. A continuación, se presenta una tabla (Nation, 2001, p. 27) que describe lo que implica conocer una palabra en la forma, el significado y el uso.

Tabla 1. Aspectos implicados en el conocimiento de una palabra

\begin{tabular}{|c|c|c|}
\hline \multirow[t]{6}{*}{ Forma } & Hablada & $\mathrm{R}_{\text {¿Cómo suena la palabra? }}$ \\
\hline & & $\mathrm{P}_{¿}$ Cómo se pronuncia? \\
\hline & Escrita & $\mathrm{R}_{¿}$ Reconozco esta palabra? \\
\hline & & $\mathrm{P}_{¿}$ Cómo se escribe o deletrea la palabra? \\
\hline & Formas morfológicas & $\begin{array}{l}\mathrm{R} \text { ¿Qué formas morfológicas son } \\
\text { reconocibles en esta palabra? }\end{array}$ \\
\hline & & $\begin{array}{l}\mathrm{P} \text { ¿Qué formas morfológicas son } \\
\text { necesarias para expresar el significado? }\end{array}$ \\
\hline \multirow[t]{3}{*}{ Significado } & Forma y significado & $\begin{array}{l}\mathrm{R} \text { ¿Qué significado indica esta forma } \\
\text { morfológica? }\end{array}$ \\
\hline & & $\begin{array}{l}P \_ \text {¿Qué forma morfológica de la palabra se } \\
\text { puede usar para expresar este significado? }\end{array}$ \\
\hline & Concepto y referentes & $\mathrm{R}$ ¿Qué se incluye en el concepto? \\
\hline
\end{tabular}




\begin{tabular}{|c|c|c|}
\hline \multirow[t]{4}{*}{ Forma } & Hablada & $\mathrm{R}_{\text {¿Cómo suena la palabra? }}$ \\
\hline & & $\begin{array}{l}\mathrm{P}_{\text {¿A } \mathrm{A} \text { qué ítems puede estar refiriéndose }} \\
\text { el concepto? }\end{array}$ \\
\hline & Asociaciones & $\mathrm{R}_{¿}$ En qué otras palabras nos hace pensar? \\
\hline & & 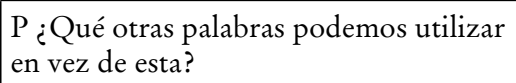 \\
\hline \multirow[t]{6}{*}{ Uso } & Funciones gramaticales & 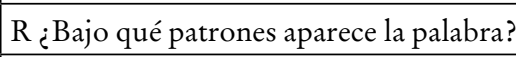 \\
\hline & & $\begin{array}{l}\mathrm{P}_{\text {¿̇Bajo qué patrones debemos usar esta }} \\
\text { palabra? }\end{array}$ \\
\hline & Colocaciones & $\begin{array}{l}\mathrm{R}_{\text {¿Qué palabras o tipos de palabras }} \\
\text { aparecen junto a ésta? }\end{array}$ \\
\hline & & $\begin{array}{l}\mathrm{P} \text { ¿Qué palabras o tipos de palabras } \\
\text { debemos usar junto a ésta? }\end{array}$ \\
\hline & $\begin{array}{l}\text { Limitaciones de uso } \\
\text { (registro, frecuencia, etc.) }\end{array}$ & $\begin{array}{l}\mathrm{R} \text { ¿Dónde, cuándo y cuan a menudo nos } \\
\text { encontramos esta palabra? }\end{array}$ \\
\hline & & $\begin{array}{l}\text { P ¿Dónde, cuándo y cuan a menudo } \\
\text { podemos usar esta palabra? }\end{array}$ \\
\hline
\end{tabular}

$\mathrm{R}$ : conocimiento receptivo; P: conocimiento productivo.

Nation (2001) usa la palabra underdeveloped (subdesarrollado) para ejemplificar el conocimiento receptivo, que se presenta en la tabla 1. Conocer la palabra underdeveloped implica, por ejemplo, ser capaz de reconocerla cuando se la escucha o lee; reconocer sus partes (o morfemas), 'under', 'develop' y 'ed'y ser capaz de relacionarlas con el significado; saber qué significa la palabra en el contexto en el que aparece y saber el concepto que subyace a la palabra que le permitirá comprenderla en una variedad de contextos. Desde el punto de vista del conocimiento productivo y su uso, conocer la palabra underdeveloped puede implicar, por ejemplo, ser capaz de pronunciarla correctamente incluyendo su acentuación; ser capaz de escribirla correctamente; ser capaz de construirla usando las partes (o morfemas) adecuadas con sus formas correctas. Ser capaz de producir la palabra para expresar el significado underdeveloped y ser capaz de producir la palabra en diferentes contextos para expresar el rango de significados de ella. 
En términos generales, comentan Ellis y Beaton (1993), pareciera ser que el aprendizaje y uso receptivo es más fácil que el aprendizaje y uso productivo, pero no está claro por qué el uso receptivo podría ser más fácil que el uso productivo. A continuación, se presentan algunas de las razones que estos autores creen podrían ayudar a explicar este fenómeno:

1. El aprendizaje productivo es más difícil porque requiere aprendizaje extra de los nuevos patrones de producción hablados o escritos. Esto será de mayor relevancia para aquellas lenguas que usan un sistema de escritura diferente al de la L1 y que poseen sonidos o combinaciones de sonidos diferentes.

2. En condiciones normales de aprendizaje de una lengua, el uso receptivo normalmente recibe mayor práctica que el uso productivo, pudiendo ser éste un factor importante cuando se da cuenta de las diferencias entre la amplitud léxica receptiva o productiva, especialmente, en mediciones globales de esta amplitud. Existe evidencia que sugiere la necesidad de la práctica específica tanto para el aprendizaje receptivo como para el productivo (DeKeyser y Sokalski 1996).

3. Ellis y Beaton (1993) sugieren que una palabra nueva del idioma extranjero en sus etapas iniciales de aprendizaje solo tiene un vínculo simple con su traducción en la lengua materna (la dirección receptiva).

4. Los aprendientes no están motivados a usar ciertos tipos de conocimiento productivo por una variedad de razones, las que incluyen el aspecto socio-cultural. Aunque algún vocabulario pueda ser conocido y eventualmente pueda ser usado productivamente, no se usa y permanece en el vocabulario pasivo del aprendiente.

\subsection{Medición de la competencia léxica}

Uno de los elementos fundamentales en el estudio de la competencia léxica de los aprendientes es la medición del conocimiento de las palabras en la lengua meta. Para estos efectos, una de las unidades de medición más útiles para el conteo de palabras y, eventualmente, 
el establecimiento del conocimiento léxico de un aprendiente es la de familia de palabras. Las 2000 familias de palabras más frecuentes del idioma inglés proveen entre un 80 y un $95 \%$ de cobertura de un texto, dependiendo de su tipo. Las 570 familias de palabras de la lista de palabras académicas ( $A W L$ en sus siglas en inglés) proveen un $4 \%$ adicional de cobertura en artículos de diarios y entre un 8,5 y un $10 \%$ en textos académicos. A partir de las 2000 familias de palabras más frecuentes del inglés, las terceras 1000 palabras más frecuentes pueden proveer cerca del $4,3 \%$ de cobertura, la que va decayendo en cada una de las siguientes 1000 familias de palabras.

En este sentido, uno de los instrumentos más ampliamente usados en la medición del vocabulario receptivo de un aprendiente de L2 (también utilizado en L1) es la prueba de niveles de vocabulario (Vocabulary Levels Test), VLT por sus siglas en inglés. El razonamiento teórico que subyace a la aplicación de este instrumento se deriva de investigaciones que muestran que la amplitud del vocabulario está directamente relacionada con la habilidad de utilizar la lengua meta de varias formas (Schmitt et al., 2001). Por ejemplo, el conocimiento de las 2000 familias de palabras más frecuentes del idioma inglés provee el grueso de los recursos léxicos necesarios para la comunicación básica diaria (Schonell, Meddleton y Shaw 1956). Las siguientes 1000 palabras proporcionan material adicional para el discurso oral y, adicionalmente, constituye el umbral que podría permitir a los aprendientes comenzar a leer textos auténticos como revistas o diarios (Nation 2001). Schmitt et al. (2001) agregan que la mayor parte de los estudios indican que el conocimiento de las 5000 familias de palabras más frecuentes del idioma inglés incluiría suficiente vocabulario para permitir a los aprendientes leer textos auténticos, aun cuando muchas palabras todavía les sean desconocidas, pudiendo inferir su significado ayudados por el contexto. Por su parte, los aprendientes con conocimiento de las 10000 familias de palabras más frecuentes de la lengua meta manejan un rango léxico amplio, tanto así que Hazenberg y Hulstijin (1996) descubrieron que este conocimiento de vocabulario podría servirles para enfrentar los desafíos de estudios universitarios en L2. 
Adicionalmente, Laufer y Nation (1999) crearon la prueba de niveles de vocabulario en su formato productivo controlado que está modelada en base a la prueba de niveles de vocabulario (conocimiento receptivo). Esta prueba utiliza los mismos niveles de frecuencia y los mismos ítems. Sin embargo, aquí los ítems no son provistos, sino elicitados en pequeñas oraciones. Para tal efecto, y con el propósito de evitar palabras no deseadas que pudieran adecuarse al contexto dado, se entregan las primeras letras de la palabra meta. Por su parte, la prueba de perfil de frecuencia léxica (Laufer y Nation 1995; Laufer 2005; Laufer 2012; Laufer 2013) es una prueba de conocimiento léxico productivo libre que muestra el porcentaje de palabras que usa un aprendiente en los diferentes niveles de frecuencia en un texto escrito. La prueba consiste en la producción de un texto de entre 200 y 400 palabras o casos (tokens) que se analizan en términos de su perfil de frecuencia léxica por medio de un programa computacional (Cobb 2002). Por ejemplo, si consideramos una composición de 200 palabras, en la que 150 pertenecen a las primeras 1000 palabras más frecuentes, 20 a las segundas 1000 , 20 a la lista de palabras académicas y 10 no están en ninguna de estas listas, convertidas estas cifras a porcentajes del total de las 200 palabras, el LFP del texto es, por tanto, 75\% - 10\% - 10\% - 5\%, respectivamente.

Laufer (2013) explica que el LFP es una medida cuantitativa del uso léxico en escritura, que puede distinguirse del conocimiento léxico. Al conocimiento se le considera información sobre una palabra que está almacenada e interconectada en el lexicón mental; por ejemplo, su forma hablada o escrita, sus propiedades gramaticales, sus propiedades semánticas y sus conexiones con otras palabras. El uso, por su parte, se refiere a la elección libre de las palabras de vocabulario que realizan los aprendientes para la comunicación del mensaje oral o escrito. Del mismo modo, el uso debe ser igualmente distinguido del conocimiento léxico activo. El conocimiento activo se asocia al habla o a la escritura e implica recuperar la forma deseada de la palabra cuando se nos pide hacerlo. Por ejemplo, cuando se nos pide traducir una palabra de la L1 a la L2. 
No obstante, el uso se asocia a la producción libre del aprendiente, como es el caso de la composición. Es deseable, comenta la autora, hacer la distinción entre el conocimiento activo y el uso ya que no todos los aprendientes que producen vocabulario infrecuente cuando se les fuerza a ello, lo hacen cuando se les permite realizar su propia elección de palabras, como es en el caso de la escritura de cartas, informes, composiciones o en presentaciones orales.

\subsection{La enseñanza de vocabulario}

En el aprendizaje de una lengua hay involucrados diferentes objetivos. Uno de ellos puede estar orientado hacia la gramática, otro al contenido, otro al desarrollo de las habilidades y, otro al aprendizaje de vocabulario. En este trabajo nos centramos en el aprendizaje y enseñanza de este último. Para ello es necesario tener en cuenta qué vocabulario vale la pena abordar y cuánto tiempo se le puede dedicar a ello. Nation (2001) sugiere que la enseñanza de vocabulario debe ser sistemática y responsable, con el propósito de asegurar el mayor beneficio para el aprendiente en relación al tiempo de la clase que se destine para ello. Plantea, además, que es útil invertir tiempo en la enseñanza de vocabulario si este es el objetivo de la clase, si el vocabulario es de alta frecuencia, si las palabras a enseñar pertenecen a un tema importante o técnico, o si estas palabras poseen partes útiles. También vale la pena enseñar vocabulario si estas palabras proveen una oportunidad para desarrollar estrategias de aprendizaje.

En esta línea, existe un grupo importante de estrategias que potencian el aprendizaje activo de una palabra a través del proceso de generación o creación. Existe un variado número de estudios que demuestran que el procesamiento generativo es un factor importante tanto en la legua materna como en la lengua meta (Joe 1995; R. Ellis 1995; Baddeley 1990; Laufer y Hulstijn 2001). El procesamiento de generación o uso creativo del vocabulario ocurre cuando a palabras que han sido aprendidas en un contexto determinado se les encuentra en un contexto distinto o se les usa de una manera diferente a las del primer contacto. La generación, o el procesamiento generativo, también pueden ser receptivos o productivos. 
Desde un punto de vista receptivo, ésta involucra el contacto con la palabra que se usa de nuevas maneras tanto en audición como en lectura. En su forma productiva, ésta implica la escritura o la comunicación oral de las nuevas palabras del vocabulario deseado y en nuevos contextos. Por ejemplo, el uso de la palabra 'cement' (encementar) en un contexto literal 'we cemented the path' (encementamos el camino), y en un contexto no literal (o figurativo) como 'we cemented our relationship with a drink' (fortalecimos nuestra relación con un trago).

Laufer y Hulstijn (2001), investigaron el efecto de lo que ellos llaman el involucramiento con la tarea en el aprendizaje de vocabulario o, en otras palabras, la carga de involucramiento o nivel de exigencia de la tarea de aprendizaje. Estos observaron que el involucramiento se veía afectado por tres rasgos en la tarea. Las descripciones de estos rasgos pueden considerarse intentos por operacionalizar los niveles de procesamiento:

- Necesidad. Esta no existe si el vocabulario meta no se necesita para cumplir con la tarea. La necesidad es moderada si la tarea requiere del vocabulario meta, por ejemplo, una tarea de completación de oraciones. La necesidad es fuerte si el aprendiente siente la necesidad del vocabulario meta, por ejemplo, una tarea de escritura creativa. Es un componente actitudinal de involucramiento.

- Búsqueda. Esta no existe si las formas de la palabra o sus significados son provistos como parte de la tarea, por ejemplo, una tarea de términos pareados. La búsqueda es moderada si el aprendiente tiene que buscar el significado de las palabras; y fuerte si el aprendiente tiene que buscar la forma para expresar el significado, pudiendo tratarse de una búsqueda mental o en el diccionario.

- Evaluación. La evaluación involucra decidir si la elección de una palabra es apropiada o no. La evaluación es moderada si se provee el contexto; y fuerte si el aprendiente tiene que generar el contexto. Por ejemplo, escoger entre las palabras apropiadas en un diccionario involucra evaluación. 
Se puede incrementar el vocabulario escrito de los aprendientes centrándose, por una parte, en la amplitud general del vocabulario $y$, por otra, en vocabulario específico para actividades o tareas específicas. En esta línea, las estrategias y técnicas que impulsan el uso productivo del vocabulario necesitan tomar en consideración los factores que se han descrito previamente.

En síntesis, los principales sustentos teóricos que se consideran en este trabajo son el concepto de familia de palabra que será utilizado como unidad de medida del conocimiento léxico receptivo, productivo y uso léxico que define en gran parte la competencia léxica del aprendiente. Del mismo modo, se reconoce la diferencia entre los tipos de conocimiento léxico, receptivo y productivo. El primero entendido como el conocimiento que permite la comprensión del ítem léxico desde la L2 a la L1. El segundo se entiende como aquel conocimiento que se demuestra ante el estímulo y no necesariamente por una decisión espontánea del aprendiente. Se le reconoce más específicamente en este estudio como el conocimiento productivo controlado, pues su utilización está siempre motivada por un estímulo lingüístico. En contraste, el uso léxico, a diferencia del conocimiento léxico, es aquel que se demuestra a través de la elección libre de vocabulario cuando el aprendiente debe producir un texto. Esta elección libre está determinada por cuán amplio es el conocimiento receptivo y cuánto de ese conocimiento receptivo está disponible para su uso activo. Esta transición, a través del crecimiento del conocimiento léxico, expresada en la habilidad escrita es la que se explora en este trabajo. Para esto se consideran las diferencias que existen entre la enseñanza léxica deliberada versus la incidental y la enseñanza productiva versus la receptiva, pues como indican estudios recientes, estas diferencias juegan un rol fundamental en el desarrollo de la competencia léxica del aprendiente.

\section{El estudio}

Esta investigación considera el diseño y desarrollo de un estudio de tipo cuantitativo, cuasi-experimental con pre y postest, que indaga la 
incidencia o efecto que pudiera tener un proceso de intervención léxica a través de estrategias y técnicas que impulsan el uso del vocabulario por un período prolongado de tiempo (dos semestres académicos).

Por tanto, el principal objetivo de este estudio es determinar el grado de incidencia que tiene la enseñanza de vocabulario por medio de estrategias de aprendizaje de vocabulario en la competencia léxica de aprendientes de inglés como lengua extranjera de nivel intermedio, pertenecientes a la carrera de pedagogía en inglés de una universidad chilena. Para dicho objetivo, se han formulado las siguientes preguntas de investigación:

- ¿Cómo incide la enseñanza del vocabulario a través de estrategias y técnicas apropiadas en la competencia léxica de los aprendientes de inglés como lengua extranjera de nivel intermedio?

- ¿Entre los tres tipos de conocimiento léxico que se abordan en este estudio, cuál de ellos muestra la mayor mejora?

Estas preguntas de investigación dieron paso a las siguientes hipótesis:

$\mathrm{H}_{0}$ : la enseñanza sistemática del vocabulario a través de estrategias y técnicas apropiadas no incide positivamente en la competencia léxica de los aprendientes de inglés como lengua extranjera.

$\mathrm{H}_{1}$ : la enseñanza sistemática del vocabulario a través de estrategias y técnicas apropiadas incide positivamente en la competencia léxica de los aprendientes de inglés como lengua extranjera.

$\mathrm{H}_{2}$ : el conocimiento léxico receptivo es el tipo de conocimiento léxico que muestra la mayor mejora.

\subsection{Metodología}

Para la implementación del estudio se definieron diferentes etapas. En una primera etapa, se aplicó el pretest a los participantes de ambos grupos de sujetos, experimental y de control, por medio de las pruebas de niveles de vocabulario VLT (receptiva y productiva controlada) y del perfil de frecuencia léxica LFP. Luego de esta primera recolección de datos, se procedió a su procesamiento y análisis. Cabe señalar que cada semestre académico consistió de 
18 semanas, de las cuales 4 semanas fueron destinadas a la recopilación de los datos. Las dos primeras para el pretest y las dos últimas para el postest. Durante las restantes 14 semanas se implementó el proceso de intervención.

En la segunda etapa, el proceso de intervención consistió en la implementación de 6 estrategias de aprendizaje léxico en el grupo experimental del estudio. Estas estrategias fueron parte de la planificación semestral del programa del curso de escritura inglesa de la carrera de pedagogía en inglés de la institución antes mencionada. Las sesiones de intervención tuvieron una duración de 25 minutos dentro de la cátedra de escritura inglesa que se realiza en una sesión de 4 horas pedagógicas a la semana. El vocabulario deseado, que es contemplado en el programa de la asignatura de manera incidental, se presentó explícitamente por medio de 4 estrategias que se desarrollaron clase a clase por parte del profesor y 2 estrategias que se desarrollaron como parte del trabajo personal del estudiante. Los participantes del grupo experimental cumplieron con todas las exigencias de la asignatura, solo que en lo referido al desarrollo léxico, se aplicó la metodología de enseñanza léxica sistemática con actividades orientadas al desarrollo de la competencia léxica de una manera intencionada. Para el grupo de control, sus clases de escritura inglesa se desarrollaron, posteriormente al pretest, como normalmente se han realizado, es decir, de manera incidental. El vocabulario en la asignatura en cuestión, como en otras de la lengua inglesa en el plan estudio, no se enseña deliberadamente, se privilegia el desarrollo de las habilidades, ya sean escritas u orales. No se planifica una enseñanza léxica a través de estrategias o técnicas que favorezcan el desarrollo del vocabulario. Se espera que los estudiantes lo aprendan a través de la lectura o motivados por su propio interés.

Cada sesión de intervención léxica duró 25 minutos. Durante este tiempo, los alumnos realizaron todas las actividades tendientes a la completación de dicha estrategia. Cada semana el profesor implementaba, de manera alternada, una de las 4 estrategias pensadas para su desarrollo en el aula. 


\title{
Las estrategias implementadas por el profesor y las realizadas por los alumnos se presentan en la siguiente tabla resumen:
}

\section{Tabla 2. Cuadro resumen de las estrategias aplicadas en el proceso de intervención}

\begin{abstract}
ESTRATEGIAS DE TRABAJO EN AULA
Parafraseo. En esta técnica, los participantes leyeron oraciones que luego debieron reescribir usando las palabras meta que le fueron provistas. El profesor modeló el uso de las palabras primero a través de ejemplos, basándose en un texto nuevo cada clase. Por ejemplo,

Everybody will be helped by the changes. (A todos les ayudarán los cambios) (benefit) (beneficiar).
\end{abstract}

El procedimiento cloze de 'segunda mano'. Esta técnica implicó que los participantes colocaran palabras previamente enseñadas en los espacios en blanco de un texto que resume el contenido de otro texto previamente estudiado. El contexto formal para las palabras era nuevo. Se guió a los aprendientes por medio de una lista de significados que habían sido dictados en su L1 y que correspondían a las palabras que completaban los espacios pero que los aprendientes primero debieron traducir a la L2.

La 'dicto-comp' y actividades relacionadas. Aquí, los aprendientes escucharon un texto nuevo dos veces y luego lo escribieron tratando de recordarlo. Se les motivó a usar las palabras meta de diferentes formas: viendo las palabras en la pizarra a medida que se escuchaba el texto o poniendo las palabras deseadas traducidas en la pizarra.

Uso de actividades de producción oral para influenciar el uso de vocabulario en la escritura. Los aprendientes llevaron a cabo una actividad de habla (entrevista grupal) diseñada especialmente para promover el uso del vocabulario deseado en forma oral usando un tema nuevo cada clase. Luego, reportaron los resultados de la tarea en forma oral a la clase. Finalmente, prepararon un informe escrito breve (no mayor a 1 página) sobre las conclusiones de la tarea oral en la clase.

ESTRATEGIAS DE TRABAJO PERSONAL

Registro temático. Cada participante escogió un tema de interés y por un período de tres semanas recolectó información sobre su tema desde diferentes fuentes como diarios, radio y televisión, libros, revistas, entrevistas, etc. Cada semana, el aprendiente informó de su progreso de manera oral a un grupo pequeño de la clase y al cabo de las tres semanas produjo un informe escrito que resumía la información obtenida hasta ese momento. Se le pidió al aprendiente incorporar deliberadamente en sus informes las palabras de vocabulario que habían sido aprendidas en el proceso de recolección de información de su tema. El resultado final fue un informe escrito completo.

Tarjetas de palabras. En esta estrategia, los participantes debían seleccionar, entre las palabras de los textos del curso, aquellas que les eran más difíciles de aprender y ponerlas en una tarjeta en conjunto con su definición o traducción. Diariamente, los participantes debían ir poniendo las palabras que ya habían aprendido al final de su mazo de cartas. Una vez aprendidas las palabras de manera receptiva (recordando su definición o significado), los participantes debían dar vuelta el mazo y aprender las palabras de manera productiva (recordando la palabra desde su definición o significado). Al cabo de dos semanas debían renovar el mazo. Como el avance en esta estrategia fue individual, se monitoreó el progreso con al menos tres mazos de 20 palabras como mínimo por semestre. 
La tercera etapa del estudio consistió en la medición de la competencia léxica de todos los participantes por medio del postest. Este consistió en la aplicación de las mismas pruebas aplicadas en el pretest en sus versiones equivalentes. Posteriormente, se procedió a procesar el corpus recolectado y a analizar los resultados estadísticamente.

\subsection{Sujetos}

Los participantes de este estudio son estudiantes de la carrera de pedagogía en inglés de una universidad chilena. Los participantes tienen un nivel intermedio de proficiencia en el idioma inglés y han cursado, en promedio, dos años y medio de estudios universitarios. Todos los estudiantes son nativos hablantes del español de Chile y ninguno de ellos ha tenido una experiencia de aprendizaje del idioma inglés en un país anglosajón. Su rango de edad fluctúa entre los 20 y 24 años en ambos géneros, con un promedio de $60 \%$ de predominancia femenina. Su procedencia socioeconómica es de clase media-baja, en promedio, de sectores predominantemente urbanos. Se tomaron dos grupos intactos (no se modificó su composición de ningún modo) de 18 alumnos y se designó al azar cuál sería el experimental y cuál el de control. El total de participantes que participaron del pretest fueron 36, sin embargo, por razones académicas (retiros y reprobaciones), los grupos se redujeron a 10 en el experimental y a 12 el de control. Por tanto, los alumnos que completaron el experimento en forma íntegra, concluyendo con el postest, fueron 22 participantes.

El acceso al uso de las tecnologías de información no fue prohibido a ninguno de los grupos, pues constituye un recurso que los estudiantes utilizan a diario y que está siempre presente en las actividades diarias de todos. De hecho, como parte de las clases de las diferentes asignaturas del plan de estudio de los participantes del estudio se incentiva su uso. Ambos grupos estuvieron expuestos a esta influencia y solo se utilizó el pre y postest para controlar las diferencias entre los grupos al final del período de tratamiento. 


\subsection{Recolección del corpus}

La recolección del corpus se hizo a través de la prueba de niveles de vocabulario en su formato receptivo y productivo, y por medio de la prueba de perfil de frecuencia léxica en la instancia de pretest y postest. Los tres instrumentos de recolección del corpus se detallan a continuación.

a) Prueba de vocabulario para el conocimiento receptivo La prueba VLT (Nation 1983 y 1990) mide el conocimiento de la forma escrita de la palabra, la conexión significado-forma y, en un grado menor, el conocimiento del concepto. Esta prueba (revisada y revalidada por Schmitt, Schmitt y Clapham 2001) mide principalmente el conocimiento descontextualizado de la palabra, aunque el ítem en cuestión aparece en un contexto simple. Este instrumento no mide el conocimiento léxico utilizado en comprensión auditiva, producción escrita u oral; solo el conocimiento léxico receptivo escrito.

Ejemplo 1. Ejemplo de ítem de la prueba VLT.

This is a vocabulary test. You must choose the right word to go with each meaning. Write the number of that word next to its meaning. Here is an example.

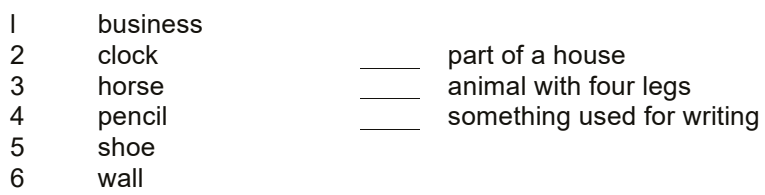

A continuación, se presenta una traducción del ejemplo 1 y las respuestas esperadas. 
Esto es una prueba de vocabulario. Usted debe elegir la palabra correcta que se ajuste a cada significado. Escriba el número de dicha palabra junto a su significado. Se presenta aquí un ejemplo.

1 negocio

2 reloj

3 caballo 6 parte de una casa

4 lápiz animal con cuatro patas

5 zapato se utiliza para escribir

6 muro

En lo relativo a la corrección de la prueba, a cada respuesta correcta se le otorga 1 punto. Cada sección está compuesta por 10 ítems de tres preguntas cada ítem. La prueba contempla 5 secciones con un puntaje total máximo de 150 puntos (ver anexo 1). Por tanto, un puntaje de 20 sobre 30 en alguno de los niveles de la prueba significa que un aprendiente conoce 667 familias de palabras de la 1000 que componen dicho nivel y, que no conoce las restantes 333. Lo deseable, según Nation (2008), es que un aprendiente conozca al menos el $90 \%$ de las familias de palabras de un nivel (27 de 30 ) para poder decir con cierta propiedad que tal nivel es conocido por el aprendiente.

b) Prueba de vocabulario para el conocimiento productivo

La prueba de conocimiento productivo controlado (Laufer y Nation, 1999) está modelada en relación a la prueba de conocimiento receptivo. Utiliza los mismos niveles de frecuencia y los mismos ítems. Sin embargo, en esta prueba los ítems no son provistos, sino elicitados en pequeñas oraciones. Para tal efecto, y con el propósito de evitar palabras no deseadas que pudieran adecuarse al contexto dado, se entregan las primeras letras de la palabra meta. Lo anterior se ilustra en el siguiente ejemplo:

Ejemplo 2. Ejemplo de ítem de la prueba VLT productiva controlada

1. They will restore the bouse to its ori state. (la respuesta esperada sería 'ginal')

(Ellos restaurarán la casa a su estado original, lit. “estado original”) 
La puntuación es en términos de correcto (1 punto) o incorrecto o en blanco ( 0 punto). Una respuesta es correcta cuando es semánticamente adecuada, esto es, se usa la palabra apropiada para expresar el significado deseado. Si se utiliza un ítem gramaticalmente incorrecto, por ejemplo, infinitivo en vez de la forma pasada, se considera correcto. Del mismo modo, una palabra mal escrita, que no distorsione el significado, por ejemplo 'recieve' en vez de 'receive', también se considera correcta. La mayoría de las palabras consideradas incorrectas incluyen pseudopalabras como ‘origan' o palabras que no correspondan al contexto. La prueba consta de un puntaje total de 90 puntos divididos en 5 secciones de 18 puntos cada una (ver anexo 2). Es importante mencionar que ambas pruebas (receptiva y productiva controlada) están disponibles en cuatro versiones paralelas equivalentes. Por tanto, cuando se aplican ambos instrumentos a los mismos sujetos o uno de ellos dos veces en pre y postest, por ejemplo; una versión diferente asegura que no reaparezcan los mismos ítems, otorgando mayor validez y confiabilidad a los datos.

c) Prueba de perfil de frecuencia léxica

La prueba de perfil de frecuencia léxica (LFP en su sigla en inglés) (Laufer y Nation 1995; Laufer 2005; Laufer 2012) es una prueba de conocimiento léxico productivo libre que muestra el porcentaje de palabras de los diferentes niveles de frecuencia que usa un aprendiente en un texto escrito. La prueba consiste en la producción de un texto de entre 200 y 400 palabras (casos) que se analizan en términos de su perfil de frecuencia léxica por medio de un programa computacional (Cobb 2002). Lo que hace el programa es cotejar las listas de frecuencia léxica con el texto que ha sido ingresado para su análisis. Para que este análisis sea llevado a cabo de una manera apropiada, se deben realizar algunas modificaciones al texto. Se deben corregir las palabras mal escritas cuyos errores no distorsionen la palabra, para que puedan ser reconocidas por el programa. Se deben omitir los nombres propios (estos no son considerados parte del léxico de un idioma en particular) y las palabras que se usaron de manera semánticamente incorrecta, por ejemplo, 
significados erróneos o mal uso de colocaciones, pues no pueden ser consideradas conocidas por los aprendientes. La composición o texto que los aprendientes deben escribir está motivada por una pregunta de ensayo y, excepto por las palabras del enunciado, no se les provee de ningún otro tipo de vocabulario.

Ejemplo 3. Pregunta de ensayo posible para la composición

- Should a government be allowed to limit the number of children in a family?

(¿Se le debería permitir a los gobiernos limitar el número de hijos por familia?)

En síntesis, la prueba VLT permitió obtener datos relativos al conocimiento léxico receptivo, por medio del reconocimiento de ítems léxicos en las diferentes bandas de frecuencia que mide esta prueba. El conocimiento productivo controlado, por su parte, se evaluó por medio de la prueba VLT en su versión productiva, que busca obtener la forma léxica deseada completando una oración de un contexto limitado y, por último, la composición de un texto escrito que nos permitió analizar el uso libre de vocabulario por medio de la prueba de Perfil de Frecuencia Léxica. La aplicación de esta batería de instrumentos permitió obtener una visión más completa de la competencia léxica de los participantes del estudio y así a bordar de mejor forma las preguntas de investigación.

\section{Los resultados}

A continuación, se presentan los resultados del pretest en las pruebas VLT receptiva, VLT productiva controlada y LFP de las composiciones que realizaron ambos grupos de sujetos. Primeramente, se estableció una comparación inicial entre los resultados de ambos grupos para establecer su comparabilidad. Como se observa en la tabla 3, los resultados indican, de acuerdo a su medias, desviaciones estándar y valores $p$, que los grupos son comparables pues no existen diferencias estadísticamente significativas en las tres mediciones. 
Tabla 3. Comparación inicial de los resultados de las pruebas VLT y LFP de los grupos de control y experimental"

\begin{tabular}{llllll}
\hline & \multicolumn{2}{c}{$\begin{array}{c}\text { Grupo Control } \\
(\mathrm{N}=12)\end{array}$} & \multicolumn{2}{c}{$\begin{array}{c}\text { Grupo Experimental } \\
(\mathrm{N}=10)\end{array}$} \\
\hline Variable & Media & D.E. & Media & D.E. & p-valor \\
\hline R_2000 & 911,11 & 114,89 & 943,33 & 56,76 & 0,4047 \\
R_3000 & 769,44 & 166,03 & 793,33 & 127,46 & 0,7137 \\
R_5000 & 763,89 & 174,34 & 736,67 & 143,54 & 0,6975 \\
R_UWL & 897,22 & 95,83 & 906,67 & 43,89 & 0,7642 \\
R_10000 & 436,11 & 129,85 & 353,33 & 198,89 & 0,2538 \\
\hline & Resultados de la prueba VLT receptiva & \\
Variable & Media & D.E. & Media & D.E. & p-valor \\
\hline PC_2000 & 861,11 & 121,95 & 888,89 & 107,98 & 0,5818 \\
PC_3000 & 541,67 & 132,16 & 544,44 & 179,16 & 0,967 \\
PC_5000 & 236,11 & 113,92 & 266,67 & 165,22 & 0,6142 \\
PC_UWL & 513,89 & 85,82 & 577,78 & 91,47 & 0,1070 \\
PC_10000 & 78,7 & 80,19 & 88,89 & 102,1 & 0,7958 \\
\hline
\end{tabular}

Resultados de la prueba VLT productiva controlada

\begin{tabular}{llllll} 
Variable & Media & D.E. & Media & D.E. & $p$-valor \\
\hline Token & 213,33 & 51,36 & 198,5 & 36,84 & 0,4544 \\
LFP_1000 & 188,33 & 47,22 & 172,1 & 33,41 & 0,3727 \\
LFP_2000 & 13,08 & 3,78 & 10,9 & 5,22 & 0,2687 \\
LFP_OFF & 6,42 & 5,79 & 6,5 & 3,03 & 0,9677 \\
LFP_1_2 & 201,42 & 48,95 & 183 & 36,26 & 0,3367 \\
LFP_UWL_OFF & 11,92 & 6,37 & 15,5 & 3,81 & 0,1350 \\
\hline
\end{tabular}

Resultados de la prueba LFP

*Resultados expresados en número de familias de palabras por banda de frecuencia.

Como se observa, ambos grupos presentan resultados similares en las tres pruebas, tanto en las bandas de frecuencia de la prueba VLT receptiva (R_2000; R_3000; R_5000; R_UWL o lista de palabras académicas y R_10000) como en la prueba VLT productiva controlada. En cuanto a la prueba de perfil de frecuencia léxica LFP, que entrega datos sobre las primeras 1000 familias de palabras 
más frecuentes (LFP_1000) y las segundas mil (LFP_2000), los resultados son muy semejantes. Similarmente, los resultados de las palabras que están fuera de las dos primeras bandas de frecuencia (LFP_OFF) y la suma de las palabras académicas (LFP_UWL) con estas últimas, muestran un comportamiento equivalente.

Los datos de las pruebas VLT receptiva y productiva controlada corresponden al número de familias de palabra que los sujetos conocen por banda de frecuencia medida con un máximo de 1000. Por ejemplo, la media del grupo controlado en R_2000 es de 911,11, lo que quiere decir que poseen un dominio de más del $90 \%$ de esta banda de frecuencia. Lo propio hace el grupo experimental con una media de 943,33 en dicha banda. En relación a la prueba LFP, los resultados están relacionados con el número de palabras que produjo cada participante. Por ejemplo, los casos (token) promedio de las composiciones fue de 213,33 palabras, de las cuales 188,33 corresponden a las primeras 1000 familias de palabras más frecuentes del idioma inglés y 13,08 a las siguientes 1000. La diferencia la componen las palabras académicas y las que están fuera de estas listas de palabras. Algo similar se observa en la distribución de los resultados en el grupo experimental, con una media de casos (token) de 198,5, de las cuales 172,1 y 10,9 corresponden a las bandas LFP 1000 y 2000 , respectivamente.

Para dar respuesta a las preguntas de investigación se compararon los resultados de los dos grupos en relación con los resultados de las medias, desviación estándar y los valores $p$ obtenidos en las tres pruebas de vocabulario del pre y postest. En la tabla 4 se resumen los resultados comparativos del antes y después del proceso de intervención en el grupo de control de la prueba VLT receptiva, en donde se observa que no hay cambios significativos, excepto una mejora en la banda R_3000. Luego de dos semestres académicos se esperaba que hubiera habido un progreso en el conocimiento léxico de los aprendientes, sin embargo, se observa que este cambio es significativo solo en una de las bandas de frecuencia. En general, el conocimiento receptivo de este grupo se mantuvo estable. 
Tabla 4. Comparación antes y después en la prueba VLT receptiva del grupo de control

\begin{tabular}{lccccc}
\hline \multicolumn{5}{c}{ Prueba VLT receptiva. Grupo control } \\
Variable & \multicolumn{2}{c}{ Antes } & \multicolumn{2}{c}{ Después } & \\
$($ N=12) & Media & D.E. & Media & D.E. & valor $p$ \\
\hline R_2000 & 911,11 & 114,89 & 938,89 & 63,3 & 0,1930 \\
R_3000 & 769,44 & 166,03 & 866,67 & 114,59 & $0,0048^{*}$ \\
R_5000 & 763,89 & 174,34 & 777,78 & 165,35 & 0,7148 \\
R_UWL & 897,22 & 95,83 & 919,44 & 59,39 & 0,2199 \\
R_10000 & 436,11 & 129,85 & 519,44 & 218,56 & 0,2134 \\
\hline
\end{tabular}

*Estadísticamente significativo

Una situación similar se da con la prueba VLT productiva controlada en la que se presentan cambios significativos en solo dos de las bandas de frecuencia, PC_5000 y PC_10000, como se observa en la tabla 5. Estos cambios positivos son esperables como parte de la evolución normal de los estudiantes en la asignatura de la que son parte (escritura inglesa). En ella, los participantes debían escribir diferentes textos, sobre diferentes tópicos y atendiendo las exigencias del programa de dicha actividad curricular, incorporando nuevos ítems léxicos y estructuras gramaticales propias del nivel superior al que van aspirando los estudiantes que cursan la carrera. De estas dos mejoras, destaca la banda de las 10000, que corresponde a familias de palabras de muy baja frecuencia (PC_10000), con un aumento de poco más de 300 familias, moderadamente superior a la del grupo experimental con cerca de 300.

En relación con los resultados de la composición libre, no solo se mantuvo sin cambios incrementales significativos, sino que además experimentó una baja en el número de familias de palabras pertenecientes a la banda 2000 (Fam_2000) y al número total de palabras en esta banda de frecuencia (LFP_2000). Esto indicaría una menor sofisticación del texto postest, aun cuando la suma de la banda 1000 y 2000 no muestra variaciones significativas, debido a una producción estable en la banda 1000 en ambos momentos. 


\section{Tabla 5. Comparación antes y después en la prueba VLT productiva controlada del grupo de control}

\begin{tabular}{llllll}
\hline & \multicolumn{4}{c}{ Prueba VLT productiva controlada. Grupo control } \\
Variable & \multicolumn{2}{c}{ Antes } & \multicolumn{2}{c}{ Después } & \\
$($ N=12) & Media & D.E. & Media & D.E. & valor $p$ \\
\hline PC_2000 & 861,11 & 121,95 & 819,44 & 172,66 & 0,3450 \\
PC_3000 & 541,67 & 132,16 & 550,93 & 205,78 & 0,8148 \\
PC_5000 & 236,11 & 113,92 & 425,93 & 166,39 & $0,0021 \%$ \\
PC_UWL & 513,89 & 85,82 & 518,52 & 222,64 & 0,9404 \\
PC_10000 & 78,7 & 80,19 & 439,81 & 181,12 & $<0,0001 \%$ \\
\hline
\end{tabular}

*Estadísticamente significativo

Tabla 6. Comparación antes y después en la prueba LFP composición libre del grupo de control

\begin{tabular}{llllll}
\hline & \multicolumn{3}{c}{ Prueba VLT productiva controlada. Grupo control } \\
Variable & \multicolumn{2}{c}{ Antes } & \multicolumn{2}{c}{ Después } & \\
(N=12) & Media & D.E. & Media & D.E. & valor $p$ \\
\hline Token & 213,33 & 51,36 & 209,67 & 43,78 & 0,7571 \\
Fam_1000 & 80,25 & 17,35 & 80,42 & 15,23 & 0,9647 \\
LFP_1000 & 188,33 & 47,22 & 186 & 38 & 0,8195 \\
Fam_2000 & 9,08 & 3,2 & 5,67 & 3,06 & $0,0013 \%$ \\
LFP_2000 & 13,08 & 3,78 & 7,25 & 4,18 & $<0,0001 \%$ \\
Fam_UWL & 4,58 & 1,88 & 5,92 & 4,17 & 0,3427 \\
LFP_UWL & 5,5 & 2,61 & 7,33 & 5,74 & 0,3559 \\
LFP_OFF & 6,42 & 5,79 & 9,08 & 4,8 & 0,0739 \\
LFP_1_2 & 201,42 & 48,95 & 193,25 & 38,65 & 0,4362 \\
LFP_UWL_OFF & 11,92 & 6,37 & 16,42 & 8,61 & 0,1015 \\
\hline
\end{tabular}

* Estadísticamente significativo.

En contraste, el grupo experimental muestra resultados incrementales significativos en todas las pruebas aplicadas, después de la aplicación del proceso de intervención léxica, destacando una mejora interesante en el uso activo del vocabulario. En cuanto a la 
prueba VLT receptiva, podemos observar que hubo mejoras significativas en las bandas R_3000, R_5000 y R_10000, siendo esta última la que más progresa. En general, se puede observar que el incremento es más transversal que el de su contraparte.

Tabla 7. Comparación antes y después en la prueba VLT receptiva del grupo experimental

\begin{tabular}{llllll}
\hline \multicolumn{5}{c}{ Prueba VLT receptiva. Grupo Experimental } \\
Variable & \multicolumn{2}{c}{ Antes } & \multicolumn{2}{c}{ Después } \\
$(\mathrm{N}=10)$ & Media & D.E. & Media & D.E. & valor $p$ \\
\hline R_2000 & 943,33 & 56,76 & 970 & 36,68 & 0,0528 \\
R_3000 & 793,33 & 127,46 & 913,33 & 80,43 & $0,0023 \%$ \\
R_5000 & 736,67 & 143,54 & 796,67 & 108,24 & $0,0266 \%$ \\
R_UWL & 906,67 & 43,89 & 940 & 53,98 & 0,0629 \\
R_10000 & 353,33 & 198,89 & 503,33 & 162,12 & $0,0139 *$ \\
\hline
\end{tabular}

* Estadísticamente significativo.

Del mismo modo, los resultados de la prueba productiva controlada muestran un incremento significativo en las bandas PC_5000 y PC_10000. En este grupo, el mayor incremento se da igualmente en la banda 10000 , lo que es consistente con las mejoras presentadas en la prueba receptiva. Variados estudios han establecido que cuando mejora el conocimiento receptivo, también lo hace el conocimiento productivo (Zhou 2010; Zhong 2016). Los resultados presentados en la tabla 8 podrían estar reforzando estos hallazgos.

En relación con los resultados de la composición libre. Es posible observar un incremento en el número total de palabra que producen los participantes del grupo experimental, lo que, a su vez, se traduce en un aumento significativo en las palabras de la banda 1000 , en las familias de palabras que utilizan esta banda y en la suma de las bandas 1000 y 2000 (LFP_1_2), que como se estableció en la sección teórica de este trabajo, constituyen alrededor del $85 \%$ de las palabras de cualquier texto. En comparación con el grupo de control, el resto de las mediciones se mantienen, no se producen bajas 


\section{Tabla 8. Comparación antes y después en la prueba VLT productiva controlada del grupo experimental.}

\begin{tabular}{lccccc}
\hline \multicolumn{5}{c}{ Prueba VLT productiva controlada. Grupo Experimental } \\
Variable $(\mathrm{N}=10)$ & Media & D.E. & Media & D.E. & valor $p$ \\
\hline PC_2000 & 888,89 & 107,98 & 850 & 94,61 & 0,3434 \\
PC_3000 & 544,44 & 179,16 & 550 & 172,63 & 0,8638 \\
PC_5000 & 266,67 & 165,22 & 483,33 & 185,28 & $<0,0001 \%$ \\
PC_UWL & 577,78 & 91,47 & 588,89 & 192,81 & 0,8439 \\
PC_10000 & 88,89 & 102,1 & 366,67 & 211,47 & $0,0006 \%$ \\
\hline
\end{tabular}

*Estadísticamente significativo.

y las mejoras se producen en cuatro indicadores. Sin embargo, es necesario observar los datos con precaución, pues los aumentos están dados según la media de los casos (token). En otras palabras, a mayor número de palabras escritas, más palabras de las bandas analizadas es posible que se encuentren en este perfil. Proporcionalmente, los resultados podrían ser similares. No obstante, el trabajo realizado en el proceso de intervención propendió a la producción de textos más extensos y de mayor sofisticación.

Aun cuando es posible observar que hubo mejoras significativas en el grupo experimental en una variedad de bandas de frecuencia en las tres pruebas aplicadas en relación con el grupo de control, es necesario analizar si las diferencias entre los dos grupos son significativas. A continuación, se presenta un análisis de las diferencias en relación con las tres mediciones (VLT receptiva, productiva controlada y LFP)

En la tabla 10 se observa que las diferencia entre ambos grupos solo son significativas en los resultados del LFP de la composición libre en varios de sus indicadores. Las diferencias de las medias en los casos (token) muestran que el grupo experimental $(38,3)$ supera ampliamente al grupo de control $(-3,66)$; este último incluso con una diferencia negativa. Algo muy similar ocurre con la banda 
Tabla 9. Comparación antes y después en la prueba LFP composición libre del grupo experimental

\begin{tabular}{llllll}
\hline \multicolumn{5}{c}{ Prueba VLT productiva controlada. Grupo Experimental } \\
Variable & \multicolumn{2}{c}{ Antes } & \multicolumn{2}{c}{ Después } \\
$(\mathrm{N}=10)$ & Media & D.E. & Media & D.E. & valor $p$ \\
\hline Token & 198,5 & 36,84 & 236,8 & 16,46 & $0,0016 \%$ \\
Fam_1000 & 75,2 & 7,16 & 88,75 & 7,37 & $0,0006 \%$ \\
LFP_1000 & 172,1 & 33,41 & 209,4 & 15,36 & $0,0002 \%$ \\
Fam_2000 & 6,4 & 2,72 & 6,45 & 2,22 & 0,9573 \\
LFP_2000 & 10,9 & 5,22 & 7,75 & 2,51 & 0,1018 \\
Fam_UWL & 7,4 & 1,58 & 7,85 & 4,36 & 0,7584 \\
LFP_UWL & 9 & 2,36 & 9,85 & 5,43 & 0,6347 \\
LFP_OFF & 6,5 & 3,03 & 9,8 & 6,78 & 0,1238 \\
LFP_1_2 & 183 & 36,26 & 217,15 & 16,34 & $0,0012 *$ \\
LFP_UWL_OFF & 15,5 & 3,81 & 19,65 & 6,73 & 0,1242 \\
\hline
\end{tabular}

*Estadísticamente significativo.

LFP_1000; a la que, como ya se ha mencionado anteriormente, pertenecen alrededor del $85 \%$ de las palabras que componen un texto. Asimismo, en el número de familias utilizadas de la banda 1000 (Fam_1000), el grupo experimental muestra un uso de 13,55 familias de palabras más que las del grupo de control $(0,17)$, lo que estaría demostrando una mayor variación léxica. También es interesante lo que ocurre con el indicador Fam_2000, que corresponde a las familias de esta banda, en la que el grupo de control decrece con un -3,41 y el grupo experimental prácticamente se mantiene inalterado, aun cuando ambos grupos caen en el número total de palabras de la banda 2000 (LFP_2000). Lo que estaría indicando, por una parte, una variación léxica mayor, y por otra, mayor sofisticación. Por último, los datos que se observan en la suma de las palabras de las bandas 1000 y 2000 (LFP_1_2) indican que el grupo de control es ampliamente superado por el experimental. Este desempeño 
indicaría que los participantes de este último grupo utilizan más familias de palabras de las primeras 1000 y segundas 1000 familias más frecuentes del idioma inglés que la del grupo de control, haciendo sus textos más léxicamente variados y sofisticados.

Tabla 10. Diferencias entre el grupo de control y experimental del pre y postest

\begin{tabular}{clll}
\hline \multicolumn{4}{c}{ Diferencias del pre y postest en ambos grupos } \\
Variable & Control & Experimental & valor $p$ \\
\hline R_2000 & 27,78 & 26,67 & 0,9644 \\
R_3000 & 97,23 & 120 & 0,5757 \\
R_5000 & 13,89 & 60 & 0,3244 \\
R_UWL & 22,22 & 33,33 & 0,6428 \\
R_10000 & 83,33 & 150 & 0,4290 \\
\hline Variable & Control & Experimental & valor $p$ \\
\hline PC_2000 & $-41,67$ & $-38,89$ & 0,9625 \\
PC_3000 & 9,26 & 5,56 & 0,9430 \\
PC_5000 & 189,82 & 216,66 & 0,6523 \\
PC_UWL & 4,63 & 11,11 & 0,9387 \\
PC_10000 & 361,11 & 277,78 & 0,2019 \\
\hline Variable & Control & Experimental & valor $p$ \\
\hline Token & $-3,66$ & 38,3 & $0,0107^{*}$ \\
Fam_1000 & 0,17 & 13,55 & $0,0098^{*}$ \\
LFP_1000 & $-2,33$ & 37,3 & $0,0045^{*}$ \\
Fam_2000 & $-3,41$ & 0,05 & $0,0095^{*}$ \\
LFP_2000 & $-5,83$ & $-3,15$ & 0,1846 \\
Fam_UWL & 1,34 & 0,45 & 0,6574 \\
LFP_UWL & 1,83 & 0,85 & 0,7110 \\
LFP_OFF & 2,66 & 3,3 & 0,7865 \\
LFP_1_2 & $-8,17$ & 34,15 & $0,0039^{*}$ \\
LFP_UWL_OFF & 4,5 & 4,15 & 0,9225 \\
\hline & & &
\end{tabular}

*Estadísticamente significativo. 
Es interesante hacer notar el desempeño del grupo experimental en la prueba VLT receptiva en relación a sus diferencias con el grupo de control. Aunque el grupo experimental no haya conseguido diferencias estadísticamente significativas con su contraparte, la tendencia en los resultados indica que el grupo de control fue superado en 4 de las 5 bandas de la prueba, destacando lo obtenido en la banda R_3000 con 22,77 familias de palabras por sobre el grupo de control, pero aún más, en la banda R_5000 en la que el grupo experimental (60) supera por más de 4 veces lo obtenido por el grupo de control $(13,89)$. Esto significa que el grupo experimental supera por 46,11 familias de palabras al grupo de control en la banda de las 5000 familias de palabras más frecuentes del idioma inglés. Más interesante aun es lo ocurrido con la banda R_10000 en la que la diferencia haciende a 66,67 familias de palabras para el grupo experimental $(150)$ por sobre el grupo de control $(83,33)$.

En cuanto a la prueba VLT productiva controlada, la tendencia es similar. Los resultados que destacan, a pesar de no ser estadísticamente significativos, son los que corresponden a la banda PC_5000 (189,82 control - 216,66 experimental), consistentes con lo obtenido en la prueba receptiva; y los de la banda de palabras académicas (PC_UWL) con 11.11 del grupo experimental por sobre los 4,63 del grupo de control. En la banda de las 10000 familias de palabras el grupo experimental es superado moderadamente por el grupo de control con 361,11 por sobre el 277,78 , respectivamente.

\section{Discusión de los resultados}

En respuesta a la pregunta de investigación uno, es posible darnos cuenta de que los resultados avalan la incidencia de una enseñanza intencional sistemática de vocabulario. Vemos que el grupo de control se mantuvo sin cambio en la mayoría de las bandas de frecuencia de las pruebas VLT receptiva y controlada; solo mejoró en una de ellas en la prueba receptiva $\left(\mathrm{R} \_3000\right)$ y en dos en la prueba productiva controlada (PC_5000 y PC_10000). En la composición libre no solo se mantuvo, sino que disminuyó en su desempeño en la banda 2000. 
Por el contrario, el grupo experimental logró mejoras significativas en tres bandas de la prueba receptiva (R_3000; R_5000 y $\mathrm{R} \_10000$ ), en dos de las bandas de la prueba productiva controlada (R_5000 y R_10000) que coinciden con las mejoras del grupo de control, y aumentó en cuatro indicadores del Perfil de Frecuencia Léxica (LFP) de la composición libre. Destacándose su aumento en el número total de palabras producidas y la suma de palabras de las bandas 1 y 2 . El resto de los indicadores se mantienen y no se muestran disminuciones, lo que tiende a probar que un tratamiento intencional y sistemático del léxico mejora no solo la sofisticación del texto a través del uso de palabras menos frecuentes, sino también la variación léxica al incorporar palabras de una misma familia haciendo uso de más tipos que casos, como lo hace el grupo experimental.

Se da cumplimiento al objetivo principal del estudio a través de las diferentes estrategias que se implementaron en el grupo experimental, que propendieron a la realización de tareas de escritura en la que los participantes incorporaran vocabulario de menor frecuencia de temas propios de la asignatura que estaban cursando, pero que, a su vez, tendían a un desarrollo global del conocimiento y uso léxico y no a un abordaje específico de un número reducido de palabras de alguna banda de frecuencia en particular. Las cuatro estrategias de trabajo en el aula incorporaban diferentes temas y textos que proveían palabras que estaban por sobre las 2000 palabras más frecuentes, integrando dos o más habilidades lingüísticas por estrategia. En esta línea, la estrategia 1 de trabajo personal del estudiante pudiera estar reforzando la contribución que hacen las estrategias del trabajo en el aula, pues en ella los alumnos abordan una variedad de temas de su elección en fuentes de información auténticas, como son los diarios, revistas especializadas, la televisión y la internet para la realización de sus informes que fueron entregados cada tres semanas y cuyo progreso fue monitoreado semanalmente. Lo propio puede estar haciendo la estrategia 2, tarjetas de palabras y la elección de las palabras de entre los textos del programa del curso para ir reforzando el aprendizaje de aquellas 
que les fueron más difíciles. Estos resultados son consistentes con los que plantean autores como Brown (19991), Groot (2000), File y Adams (2010) y Lu (2013) en conjunto con Nation (2001) y Laufer y Hulstijn (2001). En ellos se plantea el beneficio que supone la enseñanza intencionada del vocabulario por sobre el aprendizaje incidental tanto en lectura como en escritura.

Con relación a las preguntas de investigación en lo referido a la manera en que la enseñanza sistemática e intencionada incide en la competencia léxica de los aprendientes y cuál de sus variantes se desarrolla más, podemos sugerir que los resultados obtenidos por el grupo experimental son consistentes con estudios de naturaleza similar (Ellis y Beaton 1993; Laufer y Paribakht 1998; Laufer y Goldstein 2004). Es posible constatar que el conocimiento léxico, ya sea receptivo o productivo controlado se desarrolla a tasas diferentes. Mientras que el conocimiento receptivo en el grupo experimental progresó más, esta tasa fue menor en el conocimiento léxico productivo controlado. Este último y, particularmente, el uso libre se desarrollaron más lentamente. Además, es posible observar que el conocimiento receptivo fue considerablemente superior que el productivo controlado a través de las diferentes bandas de frecuencia. Si consideramos la mejora del grupo experimental en la banda R_3000, 5000 y 10000 y tomamos en cuenta las 28 semanas (14 semanas por semestre) de clases que tomó el proceso de intervención, el promedio de familias de palabras por clase es de 7,75 para la banda 3000 , sumados a las 2,14 palabras de la banda 5000 y a las 5,3 palabras de la banda 10000 , lo que hace una motivante cifra de 15,19 familias de palabras por sesión. Esto es distinto de lo obtenido por el grupo de control que solo obtiene una mejora de 3,4 palabras en todo el período pues solo subió en la banda 3000. En un estudio similar, Laufer (1998) obtuvo entre 8-9 familias de palabras al cabo de dos semestres de instrucción normal (no experimental).

En cuanto al conocimiento productivo controlado, como ya se ha dicho, su progreso se desarrolló de una manera más lenta que la del conocimiento receptivo. Sin embargo, y aun cuando las estrategias 
de enseñanza y aprendizaje aplicadas, tanto dentro como fuera del aula, propendían al desarrollo de aprendizaje productivo, los resultados muestran que el grupo experimental tuvo un desempeño solo levemente superior en la banda 5000 y uno levemente inferior en la banda 10000 que la del grupo de control. Es posible que se necesite una mayor exposición a situaciones de uso léxico, así como a mayores repeticiones para permitir un paso más efectivo y exitoso de aquel crecimiento léxico receptivo que sí se logró en el grupo experimental (Laufer y Paribakht 1998).

En relación con el uso libre de vocabulario a través de la composición y su análisis con LFP, los resultados muestran que a pesar del gran incremento en el conocimiento receptivo y lo obtenido en el conocimiento productivo controlado, los participantes no ponen este conocimiento a disposición del uso cuando se les pide que sean ellos los que hagan la elección léxica. Dicho de otro modo, es posible que el uso libre del vocabulario haya llegado a un tope, más allá del cual su desarrollo se ve dificultado. No obstante, los resultados del grupo experimental dan cuenta de una tendencia significativa al alza en el uso de las palabras pertenecientes a las primeras 1000 y segundas 1000 palabras más frecuentes (LFP_1_2), que estarían mostrando una consistente y más estable variación léxica. Por otro lado, es interesante, aunque no significativo, el resultado de la suma de las palabras académicas y las que están más allá de las 2000 palabras (LFP_UWL_OFF) que muestran una tendencia incremental en la sofisticación léxica de sus textos. Por su parte, la baja del grupo de control y el no aumento de ninguno de los indicadores del LFP pueda deberse al poco incentivo que existe para usar vocabulario más avanzado o infrecuente. Los profesores orientados a la comunicación pudieran sentirse satisfechos con la producción de textos en los que se privilegia el cumplimiento del objetivo comunicativo por sobre su riqueza léxica, y de este modo se desincentive un uso más arriesgado de palabras de mayor dificultad. 


\section{Conclusiones}

En primer lugar, es posible concluir que el grupo experimental presentó mejoras significativas en las tres mediciones aplicadas y en las diferentes bandas de frecuencias de las pruebas VLT receptiva y productiva controlada e indicadores del LFP en lo referido a la composición libre, lo que podría traducirse en una respuesta positiva a las preguntas de investigación de este estudio. La enseñanza sistemática e intencionada de vocabulario, en oposición a la incidental, sí incide positivamente en la competencia léxica de los aprendientes. Esta conclusión podría permitir rechazar la hipótesis nula $\left(\mathrm{H}_{0}\right)$ en favor de la hipótesis alternativa $\left(\mathrm{H}_{1}\right)$. Además, podemos decir que las mejoras presentadas por el grupo experimental son atribuibles al proceso de intervención, pues el grupo de control, trabajando bajo condiciones similares a la del grupo experimental, con excepción del tratamiento léxico, en su mayoría mantuvo su rendimiento en el conocimiento receptivo, en dos de las bandas productivas aumentó moderadamente y en el perfil de frecuencia léxica no solo no hubo cambios positivos, sino que disminuyó su rendimiento en dos indicadores.

No obstante, la incidencia en el desarrollo de los diferentes conocimientos y uso léxico es diferente. El conocimiento receptivo es el que más progresa en el grupo experimental, aunque este conocimiento no se traspasa fácilmente al productivo libre. En efecto, el conocimiento productivo controlado y el productivo libre se desarrollan más lentamente. Aun así, la brecha entre conocimiento receptivo y productivo controlado es más corta que la que existe entre el conocimiento receptivo y productivo libre. Este último, no obstante, presenta un desarrollo interesante en relación al grupo de control. Su variación léxica y, en menor medida, su sofisticación superan al del grupo de control ampliamente. Esto debido, principalmente, a la naturaleza productiva de las estrategias de enseñanza de vocabulario implementadas en el proceso de intervención. Nation (2001) sugiere que el conocimiento productivo se verá ampliamente favorecido por actividades de enseñanza y aprendizaje 
de tipo productivas, en otras palabras, no se puede esperar que los aprendientes desplieguen un uso efectivo del vocabulario si solamente han aprendido vocabulario de manera receptiva (por ejemplo, leyendo).

De ahí la importancia de promover un aprendizaje activo que permita que los aprendientes progresen a la siguiente fase de conocimiento a través de estrategias que los empujen a explotar todos sus recursos. En este sentido, el enfoque de la producción (output) en adición al enfoque del input es muy relevante. Si a los aprendientes se les proveyera de tareas y ejercicios, a través de diferentes estrategias, que fueran diseñadas o adaptadas específicamente a la elicitación del nuevo vocabulario que ha sido enseñado, tendrían una mayor disposición a incorporarlo en su producción libre. El uso repetido de palabras aprendidas receptivamente refuerza el conocimiento activo, pero a medida que el conocimiento receptivo aumenta, se aprenden más palabras infrecuentes. No obstante, el aprendiente puede expresar significado, comúnmente sin la necesidad de estas palabras. Si no se le empuja a usarlas puede que nunca se activen $y$, por lo tanto, se mantengan solo en el vocabulario pasivo.

\section{Referencias bibliográficas}

Albrechtsen, Dorte, Kirsten Haastrup y Birgit Henriksen

2008 Vocabulary and writing in a first and second language: processes and development. Hampshire: Palgrave Macmillan. https://doi.org/10.1057/9780230593404

Astika, Gusti G.

1993 “Analytical assessments of foreign students' writing”. RELC journal.24,61-70.https://doi.org/10.1177/003368829302400104

BADDELEY, Alan

1990 Human memory. London: Lawrence Erlbaum Associates.

Brown, Thomas y Fred Perry

1991 "A comparison of three learning strategies for ESL vocabulary acquisition”. Tesol Quarterly. 25, 4, 655-670. https://doi. org/10.2307/3587081 


\section{Centro Virtual Cervantes}

2015 Diccionario de términos clave de ELE. Consultado: 14 de diciembre de 2018. <https://cvc.cervantes.es/ensenanza/ biblioteca_ele/diccio_ele/diccionario/aprendiente.htm>.

COAdy, James

1993 "Research on ESL/EFL vocabulary acquisition: Putting it in context". En Second Language Reading and Vocabulary Learning. Eds., Thomas Huckin, Margot Haynes y James Coady. Norwood, NJ: Ablex Publishing, 3-23.

Совв, Тот

2002 Web Vocabprofile. An adaptation of Range and Frequency programs. Heatley, Nation y Coxhead 2002. Consultado: 15 de diciembre de 2017. <http://www.lextutor.ca/vp>.

Cohen, Andrew y Edna ApHek

1981 "Easifying second language learning”. Studies in Second Language Acquisition. 3, 2, 221-236. https://doi.org/10.1017/ s0272263100004198

Consejo DE Europa

2001 Marco común europeo de referencia para las lenguas: aprendizaje, enseñanza, evaluación. Madrid: Instituto Cervantes - Ministerio de Educación, Cultura y Deporte.

Dekeyser, Robert M. y Karl J. SOKALski

1996 "The differential roles of comprehension and production practice”. Language Learning. 46, 4, 613-642. https://doi. org/10.1111/j.1467-1770.2001.tb00015.x

ELLIS, Rod 1995 "Modified oral input and the acquisition of word meanings". Applied Linguistics. 16, 4, 409-441. https://doi.org/10.1093/ applin/16.4.409

Ellis, Nick y Alan BeAton

1993 "Factors affecting foreign language vocabulary: imagery keyword mediators and phonological short-term memory". The Quarterly Journal of Experimental Psychology. 46, 3, 533-558. https://doi.org/10.1080/14640749308401062 
File, Kieran y Rebecca Adams

2010 "Should vocabulary instruction be integrated or isolated?". Tesol

Quarterly. 44, 2, 222-249. https://doi.org/10.5054/tq.2010.219943

Groot, Peter J. M.

2000 "Computer assisted second language vocabulary acquisition". Language Learning \& Technology, 4, 1, 60-81.

Hazenberg, Suzanne y Jan H. Hulstun

1996 "Defining a minimal receptive second language vocabulary for non-native university students: an empirical investigation”. Applied Linguistics. 17, 2, 145-163. https://doi.org/10.1093/ applin/17.2.145

Hulstijn, Jan H., Merel Hollander y Tine Greidanus

1996 "Incidental vocabulary learning by advanced foreign language students: the influence of marginal glosses, dictionary use, and reoccurrence of unknown words". The Modern Language Journal. 80, 3, 327-339. https://doi. org/10.1111/j.1540-4781.1996.tb01614.x

Joe, Angela

1995 “Text-based tasks and incidental vocabulary learning”. Second Language Research. 11, 2, 149-158. https://doi.org/10.1177/ 026765839501100206

KoDA, Keiko

1989 "The effects of transferred vocabulary knowledge on the development of L2 reading proficiency". Foreign Language Annals. 22, 6, 529- 540. https://doi.org/10.1111/j.1944-9720.1989.tb02780.x

LAUFER, Batia

1998 "The development of passive and active vocabulary in a second language: same or different?”. Applied Linguistics. 19, 2, 255271. https://doi.org/10.1093/applin/19.2.255

2005 "Lexical frequency profile: from Montecarlo to the real world. A response to Meara”. Applied Linguistics. 26, 4, 581-587. https://doi.org/10.1093/applin/ami029

2012 "Lexical frequency profile”. En The Encyclopaedia of Applied Linguistics. Eds, Carol A. Chapelle. Boston: Wiley-Blackwell. 2013 "Vocabulary and writing". En The encyclopaedia of Applied Linguistics. Eds., Carol A. Chapelle. Boston: Wiley-Blackwell. 
LAUfer, Batia y Zahava Goldstein

2004 "Testing Vocabulary Knowledge: size, strength and computer adaptiveness”. Language Learning. 54, 3, 469-523. https://doi. org/10.1111/j.0023-8333.2004.00260.x

LAufer, Batia y Jan H. Hulstijn

2001 "Incidental vocabulary acquisition in a second language: the effect of task-induced involvement load". Applied Linguistics. 22, 1, 1-26. https://doi.org/10.1093/applin/22.1.1

LAufer, Batia y Paul Nation

1995 "Vocabulary size and use: lexical richness in L2 written production”. Applied Linguistics. 16, 3, 307-322. https://doi. org/10.1093/applin/16.3.307

1998 "The relationship between passive and active vocabularies: effects of language learning context". Language Learning. 48, 3, 365-391. https://doi.org/10.1111/0023-8333.00046

1999 "A vocabulary-size test of controlled productive ability". Language Testing. 16, 1, 33-51.

LAUfer, Batia y Sima PARIBAKHT

1998 "The relationship between passive and active vocabularies: effects of language learning context". Language Learning. 48, 3, 365-391.

LEE, SIOK

2003 "ESL learners' vocabulary use in writing and the effects of explicit vocabulary instruction”. System. 31, 4, 537-561. https://doi.org/10.1016/j.system.2003.02.004

LEKI, Ilona y Joan G.CARSON

1994 "Students' perceptions of EAP writing instruction and writing needs across the disciplines”. Tesol Quarterly. 28, 1, 81-101. https://doi.org/10.2307/3587199

Luoma, Sari

2004 Assessing speaking. Cambridge: Cambridge University Press. https://doi.org/10.1017/cbo9780511733017

Luppescu, Stuart y, Richard R. DAY

1993 "Reading dictionaries and vocabulary learning”. Language Learning. 43, 2, 263-287. https://doi.org/10.1111/j.1467-1770. 1992.tb00717.x 
LINNARUD, Moira

1986 Lexis in composition: a performance analysis of Swedish learners' written English. Lund: Gleerup.

Llach, María Pilar y Melania Gallego

2009 "Examining the relationship between receptive vocabulary size and written skills of primary school learners". Atlantis. $31,1,129-147$.

Lu, Minhui

2013 "Effects of four vocabulary exercises on facilitating learning vocabulary meaning, form and use”. Tesol Quarterly. 47, 1, 167-176. https://doi.org/10.1002/tesq.79

Meara, Paul

1990 "Some notes on the Eurocentres vocabulary size tests". Foreign language comprehension and production. 103-113.

Towell, Richard, Roger Hawkins y N. Bazergui

1996 "The development of fluency in advanced learners of French". Applied Linguistics. 17, 84-119. https://doi.org/10.1093/ applin/17.1.84

Melka-Teichroew, Francine J.

1982 "Receptive versus productive vocabulary: a survey". Interlanguage Studies Bulletin. 6, 2, 5-33.

MORRIS, Lori y Tom COBB

2004 "Vocabulary profiles as predictors of the academic performance of teaching English as a second language trainees". System. 32, 1, 75-87. https://doi.org/10.1016/j.system.2003.05.001

Nation, Paul

1983 "Testing and teaching vocabulary". Guidelines. 5, 1, 12-25.

1990 Teaching and learning vocabulary. Massachusetts: Newbury House.

2001 Learning vocabulary in another language. Cambridge: Cambridge University Press. https://doi.org/10.1017/CBO9781139858656

2008 Teaching Vocabulary: strategies and techniques. Boston: Heinle Cengage Learning.

Nation, Paul y Paul Meara

2010 "Vocabulary". En An introduction to Applied Linguistics. Eds., Norbert Schmitt. New York: Edward Arnold, 34-52. 
Palmberg, Rolf

1987 "Patterns of vocabulary development in foreign language learners". Studies in Second Language Learning. 9, 2, 201-220. https://doi.org/10.1017/s0272263100000474

Polio, Charlene y Margo GLEw

1996 "ESL writing assessment prompts: How students choose". Journal of Second Language Writing. 5, 1, 35-49. https://doi. org/10.1016/s1060-3743(96)90014-4

READ, John

1993 "The development of a new measure of L2 vocabulary knowledge”. Language Testing, 10, 355-371. https://doi.org/ 10.1177/026553229301000308

2000 Assessing vocabulary. Cambridge: Cambridge University Press. https://doi.org/10.1017/cbo9780511732942

Schmitt, Norbert, Diane Schmitt y Caroline Clapham

2001 "Developing and exploring the behaviour of two new versions of the vocabulary levels test”. Language Testing. 18, 1, 55-88. https://doi.org/10.1191/026553201668475857

Schonell, Fred, I. Meddleton y B. Shaw

1956 A study of the oral vocabulary of adults. Brisbane: University of Queensland Press.

YOSHIDA, Midori

1978 "The acquisition of English vocabulary by a Japanese speaking child”. En Second Language Acquisition: a Book of Readings. Eds., Evelyn Hatch. Rowly. MA: Newbury House, 91-100.

ZHONG, H. F.

2016 "The relationship between receptive and productive vocabulary knowledge: A perspective from vocabulary use in sentence writing”. The Language Learning Journal, 1-17. https://doi. org/10.1080/09571736.2015.1127403

Zноu, S.

2010 "Comparing receptive and productive academic vocabulary knowledge of Chinese EFL learners". Asian Social Science. 6,10, 14-19. https://doi.org/10.5539/ass.v6n10p14 


\section{Anexo 1. Prueba VLT conocimiento receptivo}

A vocabulary levels test: Version 1

This is a vocabulary test. You must choose the right word to go with each meaning. Write the number of that word next to its meaning. Here is an example.

$\begin{array}{ll}1 & \text { business } \\ 2 & \text { clock } \\ 3 & \text { horse } \\ 4 & \text { pencil } \\ 5 & \text { shoe } \\ 6 & \text { wall }\end{array}$

part of a house animal with four legs something used for writing

You answer it in the following way.

$\begin{array}{ll}1 & \text { business } \\ 2 & \text { clock } \\ 3 & \text { horse } \\ 4 & \text { pencil } \\ 5 & \text { shoe } \\ 6 & \text { wall }\end{array}$

part of a house

animal with four legs

something used for writing

Some words are in the test to make it more difficult. You do not have to find a meaning for these words. In the example above, these words are business, clock, shoe.

Try to do every part of the test.

The 2,000 word level

1 birth

2 dust

3 operation game

4 row winning

5 sport being born

6 victory

1 choice

2 crop heat

3 flesh meat 
4 salary

5 secret money paid regularly for doing a job

6 temperature

1 cap

2 education teaching and learning

3 journey numbers to measure with

4 parent

5 scale going to a far place

6 trick

1 attack

2 charm

3 lack

4 pen

5 shadow

6 treasure

1 cream

2 factory part of milk

3 nail a lot of money

4 pupil

5 sacrifice person who is studying

6 wealth

1 adopt

$2 \mathrm{climb}$

3 examine

4 pour

5 satisfy

6 surround

1 bake

2 connect

3 inquire gold and silver pleasing quality not having something

4 limit

5 recognize

6 wander

1 burst

2 concern break open

3 deliver make better

4 fold go up look at closely be on every side

5 improve take something to someone

6 urge 
1 original

2 private

3 royal

4 slow

5 sorry

6 total

1 brave

2 electric commonly done

3 firm wanting food

4 hungry having no fear

5 local

6 usual

first not public all added together

The 3,000 word level

1 belt

2 climate

3 executive

4 notion

5 palm

6 victim

1 acid

2 bishop

3 chill

4 ox

5 ridge

6 structure

1 bench

2 charity

3 jar

4 mate

5 mirror

6 province

1 boot

2 device

3 lieutenant army officer

4 marble cold feeling farm animal inner surface of your hand strip of leather worn around the waist

5 phrase a kind of stone

6 vein tube through which blood flows 
1 apartment

2 candle a place to live

$3 \mathrm{draft}$ chance of something happening

4 horror first rough form of something written

5 prospect

6 timber

1 betray

2 dispose

3 embrace frighten

4 injure

5 proclaim

6 scare

1 encounter

2 illustrate meet

3 inspire

4 plead

5 seal say publicly hurt seriously

6 shift

1 assist

2 bother

3 condemn help

4 erect

5 trim beg for help close completely

6 whirl

1 annual

2 concealed wild

3 definite clear and certain

4 mental happening once a year

5 previous

6 savage

$1 \mathrm{dim}$

2 junior

3 magnificent wonderful cut neatly spin around quickly

4 maternal

5 odd not clearly lit

6 weary 
The 5,000 word level

1 balloon

2 federation bucket

3 novelty unusual interesting thing

4 pail rubber bag that is filled with air

5 veteran

6 ward

1 alcohol

2 apron

3 hip stage of development

4 lure state of untidiness or dirtiness

5 mess cloth worn in front to protect your clothes

6 phase

1 apparatus

2 compliment expression of admiration

3 ledge set of instruments or machinery

4 revenue money received by the government

5 scrap

6 tile

1 bulb

2 document female horse

3 legion large group of soldiers or people

4 mare a paper that provides information

5 pulse

6 tub

1 concrete

2 era

3 fibre circular shape

4 loop top of a mountain

5 plank a long period of time

6 summit

1 blend

2 devise mix together

3 hug

4 lease plan or invent

5 plague hold tightly in your arms

6 reject 
1 abolish

2 drip bring to an end by law

3 insert

4 predict guess about the future

5 soothe calm or comfort someone

6 thrive

1 bleed

2 collapse come before

3 precede fall down suddenly

4 reject move with quick steps and jumps

5 skip

6 tease

1 casual

2 desolate sweet-smelling

3 fragrant only one of its kind

4 radical good for your health

5 unique

6 wholesome

1 gloomy

2 gross

3 infinite empty

$4 \operatorname{limp}$ dark or sad

$5 \mathrm{slim}$ without end

6 vacant

Academic Vocabulary

1 benefit

2 labour work

3 percent part of 100

4 principle general idea used to guide one's actions

5 source

6 survey

1 element

2 fund

3 layer

4 philosophy 5 proportion money for a special purpose skilled way of doing something

6 technique study of the meaning of life 
1 consent

2 enforcement total

3 investigation agreement or permission

4 parameter trying to find information about something

5 sum

6 trend

1 decade

2 fee 10 years

3 file

4 incidence

5 perspective subject of a discussion money paid for services

6 topic

1 colleague

2 erosion

3 format action against the law

4 inclination

5 panel

6 violation

1 achieve

2 conceive

3 grant

4 link

5 modify

6 offset

1 convert

2 design

3 exclude keep out

4 facilitate

5 indicate wearing away gradually shape or size of something

6 survive

1 anticipate

2 compile

3 convince

4 denote change connect together finish successfully

5 manipulate

6 publish stay alive change from one thing into another

1 equivalent 2 financial most important 
3 forthcoming ___ concerning sight

4 primary concerning money

5 random

6 visual

1 alternative

2 ambiguous

3 empirical last or most important

4 ethnic

5 mutual something different that can be chosen

6 ultimate concerning people from a certain nation

The 10,000 word level

1 antics

2 batch foolish behaviour

3 connoisseur a group of things

4 foreboding person with a good knowledge of art or music

5 haunch

6 scaffold

1 auspices

2 dregs

3 hostage

4 jumble

5 saliva

6 truce

1 casualty

2 flurry

3 froth

4 revelry

5 rut

6 seclusion

1 apparition

2 botany ghost

3 expulsion confused mixture natural liquid present in the mouth worst and most useless parts of anything

4 insolence

5 leash someone killed or hurt being away from other people noisy and happy celebration

6 puddle 
1 arsenal

2 barracks happiness

3 deacon difficult situation

4 felicity minister in a church

5 predicament

6 spore

1 acquiesce

2 bask to accept without protest

3 crease

4 demolish sit or lie enjoying warmth

5 overhaul make a fold on cloth or paper

6 rape

1 blaspheme

2 endorse slip or slide

3 nurture give care and food to

4 skid speak badly about God

5 squint

6 straggle

1 clinch

2 jot

3 mutilate

4 smoulder

5 topple

6 whiz

1 auxiliary

2 candid

3 luscious

4 morose

5 pallid

6 pompous

1 dubious

2 impudent move very fast injure or damage burn slowly without flame

3 languid

4 motley

5 opaque

bad-tempered full of self-importance helping, adding support

6 primeval rude very ancient of many different kinds 


\section{Anexo 2. Prueba VLT conocimiento productivo controlado}

\section{Productive Vocabulary Levels \\ Test Version A}

Complete the underlined words as in the following example.

$\mathrm{He}$ was riding a bi.

He was riding a bicycle.

\section{THE 2,000 WORD LEVEL}

1 They will restore the house to its orig state.

2 My favourite spo is football.

3 Each room has its own priv bath and WC.

4 The tot number of students at the university is 12,347 .

5 They met to ele a president.

6 Many companies were manufac computers.

7 In AD 636 an Arab army won a famous vict over another army.

8 The lakes become ice-free and the snow mel

9 They managed to steal and hi some knives.

10 I asked the group to inv her to the party.

11 She shouted at him for spoi her lovely evening.

12 You must spend less until your deb are paid.

13 His mother looked at him with love and pri

14 The wind roa through the forest.

15 There was fle and blood everywhere.

16 She earns a high sal as a lawyer.

17 The sick child had a very high tempe

18 The bir of her first child was a difficult time for her.

\section{THE 3,000 WORD LEVEL}

1 They need to spend less on adminis and more on production.

2 He saw an ang from Heaven.

3 The entire he of goats was killed.

4 Two old men were sitting on a park ben and talking.

5 She always showed char towards those who needed help.

6 He had a big house in the Cape Prov. 
7 Oh Harold darl , I am sorry. I did not mean to upset you.

8 Judy found herself listening to the last ec of her shoes on the hard floor.

9 He cut three large sli of bread.

$10 \mathrm{He}$ sat in the shade beneath the pa trees.

11 He had a crazy sch for perfecting the world.

12 They get a big thr out of car-racing.

13 At the beginning of their journey they encoun an English couple.

14 Nothing illus his selfishness more clearly than his behaviour to his wife.

15 He took the bag and tos it into the bushes.

16 Every year she looked forward to her ann holiday.

17 There is a defi date for the wedding.

18 His voice was loud and sav , and shocked them all to silence.

\section{THE 5000-WORD LEVEL}

1 Some people find it difficult to become independent. Instead they prefer to be tied to their mother's ap strings.

2 After finishing his degree, he entered upon a new ph in his career.

3 The workmen cleaned up the me before they left.

4 On Sunday, in his last se in Church, the priest spoke against child abuse.

5 I saw them sitting on $\underline{s t}$ at the bar drinking beer.

6 Her favorite musical instrument was a tru.

7 The building is heated by a modern heating appa.

8 He received many com on his dancing skill.

9 The government raised extra rev through tax..

10 At the bottom of a blackboard there is a le for chalk.

11 After falling off his bicycle, the boy was covered with bru.

12 The child was holding a doll in her arms and hu it.

13 We'll have to be inventive and de a scheme for earning more money.

14 The picture looks nice; the colours bl really well.

15 Nuts and vegetables are considered who food.

16 The garden was full of fra flowers.

17 Many people feel depressed and gl about the future of the mankind.

18 She ski happily down the path.

\section{THE UNIVERSITY WORD LIST LEVEL}

1 The afflu of the western world contrasts with the poverty in other parts.

2 The book covers a series of isolated epis from history.

3 Farmers are introducing innova that increase the productivity per worker.

4 They are suffering from a vitamin defic 
5 There is a short term oscill of the share index.

6 They had other means of acquiring wealth, pres , and power.

7 The parts were arranged in an arrow-head configu

8 The learners were studying a long piece of written disco

9 People have proposed all kinds of hypot about what these things are.

10 The giver prefers to remain anony

11 The elephant is indig to India.

12 You'll need a mini deposit of $\$ 20,000$.

13 Most towns have taken some eleme civil defence precautions.

14 The presentation was a series of sta images.

15 This action was necessary for the uli success of the revolution.

16 He had been expe from school for stealing.

17 The lack of money depressed and frust him.

18 The money from fruit-picking was a suppl to their regular income.

\section{THE 10000-WORD LEVEL}

1 He wasn't serious about art. He just $\underline{\mathrm{da}}$ in it.

2 Her parents will never acq to such an unsuitable marriage.

3 Pack the dresses so that they won't cre.

4 Traditionally, men were expected to nu women and children.

5 Religious people would never bl against God.

6 The car sk on the wet road.

7 The politician delivered an arrogant and pom speech.

8 The Romans used to hire au troops to help them in their battles.

9 At the funeral, the family felt depressed and mo

$10 \mathrm{His} \mathrm{pu}$ little arms and legs looked pathetic.

$11 \mathrm{~A}$ vol person will change moods easily.

12 The debate was so long and tedious that it seemed int

13 Drink it all and leave only the dre.

14 A hungry dog will sa at the smell of food.

15 The girl's clothes and shoes were piled up in a ju on the floor.

16 Some monks live apart from society in total sec

17 The enemy suffered heavy cas in the battle.

18 When the Xmas celebrations and rev ended, there were plenty of drunk people everywhere. 